[19] M. Spong and M. Vidyasagar, Robot Dynamics and Control. New York: Wiley, 1989.

[20] J. T. Spooner and K. M. Passino, "Adaptive control of a class of decentralized nonlinear systems," IEEE Trans. Automat. Contr., vol. 41, pp. 280-284, 1996

[21] D. Swaroop and J. K. Hedrick, "String stability of interconnected systems," IEEE Trans. Automat. Contr., vol. 41, pp. 349-357, 1996.

[22] M. Takegaki and G. Arimoto, "A new feedback method for dynamic control of manipulators," ASME J. Dynam. Syst. Meas., Contr., vol. 102 pp. 119-125, 1981.

[23] Y. Tang and G. Guerrero, "Decentralized robust control of robots," in Proc. American Control Conf., Philadelphia, PA, 1998.

[24] Y. Tang and M. Tomizuka, "Decentralized robust control of a class of nonlinear systems," in Proc. American Control Conf., Albuquerque, NM, 1997.

\section{Finite Settling Time Control of the Double Integrator Using a Virtual Trap-Door Absorber}

\author{
Robert T. Bupp, Dennis S. Bernstein, Vijaya S. Chellaboina, and
} Wassim M. Haddad

\begin{abstract}
Finite settling time control of the double integrator is considered. The approach taken is to design a compensator based on a virtual lossless absorber which is tuned so that, at some predetermined time, the virtual subsystem possesses all of the system's energy. At this time the controller is turned off, and the double integrator remains at rest at the origin. This strategy gives the appearance of instantaneously removing all of the system's energy as if a trap door had been sprung. A practically useful feature of the virtual trap-door absorber is that only position measurement is required. Parameters for the virtual trap-door absorber controller are chosen, and the resulting controller is compared to the classical minimal-time and minimal-energy controllers, which require measurements of both position and velocity.
\end{abstract}

Index Terms-Double integrator, finite settling time control, minimal-time and minimal-energy optimal control, virtual absorber.

\section{INTRODUCTION}

Exponential stabilization of rigid-body translational or rotational motion, $M \ddot{q}=u$, the double integrator problem, is obtained by setting $u=-a \dot{q}-b q$, where $a, b>0$. However, it is often desirable to stabilize the motion in finite time. Several approaches have been considered for this problem [1]-[6] including the classical minimal-time and minimal-energy controllers [7], [8].

The purpose of this paper is to develop a continuous-time feedback controller that achieves finite settling time for the double integrator using position measurement only. Position feedback may be practical when position information is obtained from sensors such as LVDT's, capacitive sensors, and encoders.

Manuscript received January 9, 1996; revised October 21, 1998 and September 1, 1999. Recommended by Associate Editor, O. Egeland. This work was supported in part by the Air Force Office of Scientific Research under Grants F49620-95-1-0019 and F49620-93-1-0502 and in part by the National Science Foundation under Grant ECS-9496249.

R. T. Bupp and D. S. Bernstein are with the Department of Aerospace Engineering, The University of Michigan, Ann Arbor, MI 48109-2118 USA (e-mail: rbupp@umich.edu; dsbaero@umich.edu).

V. S. Chellaboina and W. M. Haddad are with the School of Aerospace Engineering, Georgia Institute of Technology, Atlanta, GA 30332-0150 USA (e-mail: vc19@cad.gatech.edu; wm.haddad@aerospace.gatech.edu).

Publisher Item Identifier S 0018-9286(00)04096-4.
The controller we develop in this paper is based upon physical principles rather than optimality criteria. Inspired by mechanical absorbers [9], this controller emulates the action of a proof-mass absorber by applying forces that a physical absorber would apply. Since the proof-mass absorber is emulated and is not a physical device, our controller can be viewed as a virtual absorber [10]. A general treatment of controllers that exploit energy transfer between real and virtual subsystems is given in [11] and [12].

The controller design involves choosing the values of the virtual proof mass and stiffness so that, at a predetermined time, the energy of the double integrator is completely transferred to the absorber subsystem. Ordinarily, the absorber subsystem would possess all of the energy only instantaneously, after which time energy would return to the plant. However, since the time at which total energy transfer occurs is known independent of the initial state of the plant, we turn off the controller at that instant, so that all of the energy is instantaneously removed from the plant, as if it had exited through a trap door, and thus the mass subsequently remains at rest at the origin.

A virtual controller using acceleration measurements and similar to the trap door absorber is given in [13]. The use of resetting as in the present paper and [11] in order to instantaneously remove controller energy is not discussed in [13].

\section{SYSTEM DESCRIPTION}

Consider the double integrator described by

$$
M \ddot{q}_{1}=u
$$

with initial conditions $q_{1}(0)=q_{10}, \dot{q}_{1}(0)=\dot{q}_{10}$. Our goal is to use feedback control to bring the position $q_{1}(t)$ and velocity $\dot{q}_{1}(t)$ to zero in finite time using only a measurement of the position $q_{1}(t)$. The feedback controller we consider emulates the lossless system shown in Fig. 1, where the springs $K$ and $k$ as well as the mass $m$ are virtual elements whose effect on the mass $M$ is implemented by means of a dynamic compensator and a force actuator. The dynamics of the closed-loop system are given by (1) and

$$
\begin{aligned}
m \ddot{q}_{2}+k q_{2}-k q_{1} & =0 \\
u & =k q_{2}-(K+k) q_{1}
\end{aligned}
$$

where $q_{2}$ is the position of the virtual mass $m$. As shown in Fig. 2, (1)-(3) can be represented as the single-input/single-output (SISO) feedback interconnection of the double integrator plant with a second-order, proper dynamic compensator whose input is the position of the mass $M$.

The virtual absorber shown in Fig. 1 can also be applied to the harmonic oscillator. Suppose that the mass is attached to the ground by means of a stiffness with spring constant $K^{\prime}$. Then $K^{\prime}$ can be subsumed by the virtual spring constant $K$ in the analysis given below.

For notational convenience, we define the quantities

$$
\begin{aligned}
& x_{1} \triangleq K q_{1}, \quad x_{2} \triangleq K q_{2}, \quad x_{r} \triangleq x_{2}-x_{1} \\
& \kappa \triangleq k / K, \quad \tau \triangleq \sqrt{\frac{K}{M}} t, \quad \omega_{a} \triangleq \sqrt{\frac{k / m}{K / M}} .
\end{aligned}
$$

With this notation, (1)-(3) become

$$
\begin{aligned}
\ddot{x}_{1} & =u \\
\ddot{x}_{2}+\omega_{a}^{2} x_{r} & =0 \\
u & =\kappa x_{r}-x_{1}
\end{aligned}
$$




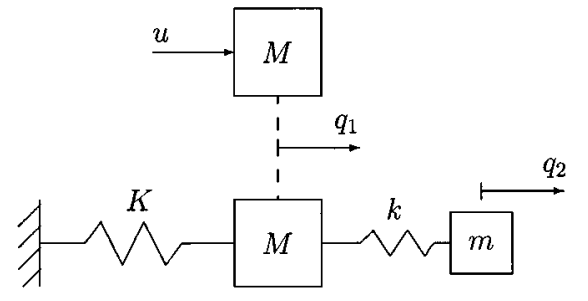

Fig. 1. The double integrator without (above) and with (below) the virtual absorber subsystem.

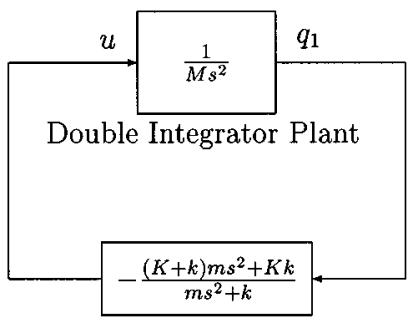

Absorber Controller

Fig. 2. Feedback control of the double integrator using the absorber controller with position feedback.

where $\left({ }^{\circ}\right)$ now represents differentiation with respect to normalized time $\tau$. The closed-loop system (4)-(6) has the form

$$
\begin{gathered}
\dot{x}=\mathcal{A} x, \quad x=\left[\begin{array}{l}
x_{1} \\
\dot{x}_{1} \\
x_{r} \\
\dot{x}_{2}
\end{array}\right] \\
\mathcal{A}=\left[\begin{array}{rrrr}
0 & 1 & 0 & 0 \\
-1 & 0 & \kappa & 0 \\
0 & -1 & 0 & 1 \\
0 & 0 & -\omega_{a}^{2} & 0
\end{array}\right] .
\end{gathered}
$$

The characteristic equation of $\mathcal{A}$ is given by $s^{4}+\left(1+\kappa+\omega_{a}^{2}\right) s^{2}+\omega_{a}^{2}=$ 0 , which can be factored as $\left(s^{2}+\omega^{2}\right)\left(s^{2}+\Omega^{2}\right)=0$, where

$$
\begin{aligned}
& \omega=\sqrt{\frac{1}{2}\left(1+\kappa+\omega_{a}^{2}\right)-\frac{1}{2} \sqrt{\left(1+\kappa+\omega_{a}^{2}\right)^{2}-4 \omega_{a}^{2}}} \\
& \Omega=\sqrt{\frac{1}{2}\left(1+\kappa+\omega_{a}^{2}\right)+\frac{1}{2} \sqrt{\left(1+\kappa+\omega_{a}^{2}\right)^{2}-4 \omega_{a}^{2}}}
\end{aligned}
$$

and the eigenvalues of $\mathcal{A}$ are $\lambda_{1,2}= \pm \jmath \omega, \lambda_{3,4}= \pm \jmath \Omega$. The closed-loop system (7) is thus Lyapunov stable. Since $\left(1+\kappa+\omega_{a}^{2}\right)^{2}-4 \omega_{a}^{2}=\left(1+\kappa-\omega_{a}^{2}\right)^{2}+4 \kappa \omega_{a}^{2}>0$, it is clear that expressions in (8) are well defined. Furthermore, note that

$$
\omega \Omega=\omega_{a}, \quad \omega^{2}+\Omega^{2}=1+\kappa+\omega_{a}^{2} .
$$

Next, we derive an expression for the time history of the state $x_{1}$ due to an initial condition of the form $x_{0}=\left[\begin{array}{llll}x_{10} & \dot{x}_{10} & 0 & 0\end{array}\right]^{T}$, or, equivalently in the dimensional states

$$
\begin{array}{llrl}
q_{1}(0) & =q_{10}, & \frac{d q_{1}}{d t}(0) & =\dot{q}_{10} \\
q_{2}(0) & =q_{10}, & \frac{d q_{2}}{d t}(0) & =0
\end{array}
$$

which corresponds to an arbitrary initial position $q_{10}=(1 / K) x_{10}$ and an arbitrary initial velocity $\dot{q}_{10}=(1 / \sqrt{K M}) \dot{x}_{10}$ of the mass $M$, with zero initial elongation of the virtual spring $k$ and zero initial velocity of the virtual mass $m$.

Taking the Laplace transform of (7) gives

$$
\begin{aligned}
X_{1}(s)= & \frac{s\left(s^{2}+\omega_{a}^{2}+\kappa\right)}{s^{4}+\left(1+\kappa+\omega_{a}^{2}\right) s^{2}+\omega_{a}^{2}} x_{10} \\
& +\frac{s^{2}+\omega_{a}^{2}}{s^{4}+\left(1+\kappa+\omega_{a}^{2}\right) s^{2}+\omega_{a}^{2}} \dot{x}_{10}
\end{aligned}
$$

which yields

$$
\begin{aligned}
x_{1}(\tau)= & x_{10}\left[\frac{1}{2}\left(1+c_{1}\right) \cos \omega \tau+\frac{1}{2}\left(1-c_{1}\right) \cos \Omega \tau\right] \\
& +\dot{x}_{10}\left[\frac{1}{2 \omega}\left(1+c_{2}\right) \sin \omega \tau+\frac{1}{2 \Omega}\left(1-c_{2}\right) \sin \Omega \tau\right]
\end{aligned}
$$

where

$$
\begin{aligned}
& c_{1} \triangleq \frac{\omega_{a}^{2}-1+\kappa}{\sqrt{\left(1+\kappa+\omega_{a}^{2}\right)^{2}-4 \omega_{a}^{2}}} \\
& c_{2} \triangleq \frac{\omega_{a}^{2}-1-\kappa}{\sqrt{\left(1+\kappa+\omega_{a}^{2}\right)^{2}-4 \omega_{a}^{2}}} .
\end{aligned}
$$

\section{Finite Settling Time Controller Synthesis}

Theorem 3.1: Let $n$ and $p$ be nonnegative integers, and choose $K$, $k, m>0$ such that

$$
\frac{k}{K}=\frac{m}{M}=\frac{4(2(p-n)+1)^{2}}{(4 n+1)(4 p+3)} .
$$

Then the solution of (1)-(3) with initial conditions (10) satisfies

$$
q_{1}\left(t_{s}\right)=0, \quad \dot{q}_{1}\left(t_{s}\right)=0
$$

where

$$
t_{s}=\frac{\pi}{2} \sqrt{(4 n+1)(4 p+3) M / K} .
$$

Furthermore, the control force $u(t)$ given by (6) is bounded by

$$
|u(t)| \leq \sqrt{(K+k)\left(K q_{10}^{2}+M \dot{q}_{10}^{2}\right)}, \quad t \geq 0 .
$$

Remark 3.1: If $K, k$, and $m$ satisfy (14), then $q_{1}(t)$ is given by

$$
\begin{aligned}
q_{1}(t)= & \frac{1}{\omega+\Omega}\left\{q_{10}\left[\Omega \cos \sqrt{\frac{K}{M}} \omega t+\omega \cos \sqrt{\frac{K}{M}} \Omega t\right]\right. \\
& \left.+\dot{q}_{10} \sqrt{\frac{M}{K}}\left[\sin \sqrt{\frac{K}{M}} \omega t+\sin \sqrt{\frac{K}{M}} \Omega t\right]\right\}, \quad t \geq 0
\end{aligned}
$$

where $\omega=1 / \Omega=\sqrt{4 n+1 / 4 p+3}$.

Remark 3.2: Note that $t_{s}$ is independent of $q_{10}$ and $\dot{q}_{10}$. Also, the smallest value of $t_{s}$ for which $q_{1}\left(t_{s}\right)=0$ and $\dot{q}_{1}\left(t_{s}\right)=0$ is obtained with $n=p=0$ in (16), which yields $t_{s}=(\pi / 2) \sqrt{3 M / K}$. This value is achieved for $k=4 K / 3$ and $m=4 M / 3$. Furthermore, $t_{s}$ can 
be made arbitrarily small by choosing $K$ sufficiently large, although large $K$ increases the control amplitude as suggested by (17).

The virtual trap-door absorber is based on Theorem 3.1. The controller in Fig. 2 is implemented for $0 \leq t \leq t_{s}$ so that, at time $t=t_{s}$, (15) holds. At this time, the controller is shut off, and thus the mass $M$ remains at rest at the origin. For the double-integrator plant written in state-space form as

$$
\dot{q}=A q+B u, \quad y=C q
$$

where

$$
\begin{aligned}
q & =\left[\begin{array}{l}
q_{1} \\
\dot{q}_{1}
\end{array}\right], \quad A=\left[\begin{array}{ll}
0 & 1 \\
0 & 0
\end{array}\right] \\
B & =\left[\begin{array}{c}
0 \\
1 / M
\end{array}\right], \quad C=\left[\begin{array}{ll}
1 & 0
\end{array}\right]
\end{aligned}
$$

the resulting linear time-varying dynamic controller has the form

$$
\begin{aligned}
& \dot{x_{c}}(t)=A_{c} x_{c}(t)+B_{c} y(t) \\
& u(t)=C_{c}(t) x_{c}(t)+D_{c}(t) y(t)
\end{aligned}
$$

where

$$
\begin{aligned}
A_{c} & =\left[\begin{array}{cc}
0 & 1 \\
-k / m & 0
\end{array}\right], \quad B_{c}=\left[\begin{array}{c}
0 \\
k / m
\end{array}\right] \\
C_{c}(t) & = \begin{cases}{\left[\begin{array}{ll}
k & 0
\end{array}\right],} & t \in\left[0, t_{s}\right) \\
{\left[\begin{array}{ll}
0 & 0
\end{array}\right],} & t \geq t_{s}\end{cases} \\
D_{c}(t) & = \begin{cases}-K-k, & t \in\left[0, t_{s}\right) \\
0, & t \geq t_{s} .\end{cases}
\end{aligned}
$$

Remark 3.3: If $M$ is not known exactly, or if some damping is present, or if some external disturbance is present, then the double integrator is not expected to reach the origin at time $t_{s}$. In this case the controller can be reset and restarted periodically with period $t_{s}$.

\section{PERformance ANALYSIS}

We first consider the full-state classical minimal-time controller given by [7] and [8]

$$
u(t)=\left\{\begin{array}{c}
-u_{\max } \operatorname{sgn}\left(\dot{q}_{1}(t)+\operatorname{sgn}\left(q_{1}(t)\right) \sqrt{2\left|q_{1}(t)\right| \frac{u_{\max }}{M}}\right) \\
\dot{q}_{1}(t)+\operatorname{sgn}\left(q_{1}(t)\right) \sqrt{2\left|q_{1}(t)\right| u_{\max } / M} \neq 0 \\
-u_{\max } \operatorname{sgn}\left(q_{1}(t)\right), \\
\dot{q}_{1}(t)+\operatorname{sgn}\left(q_{1}(t)\right) \sqrt{2\left|q_{1}(t)\right| u_{\max } / M}=0
\end{array}\right.
$$

which is characterized by a discontinuous control force $u(t)$ that switches between $\pm u_{\max }$.

Next, for a specified settling time $t_{s}$, we consider the full-state minimal-energy controller given in open-loop form by [7] and [8]

$$
\begin{gathered}
u(t)=-B^{T} e^{A^{T}\left(t_{s}-t\right)}\left(\int_{0}^{t_{s}} e^{A s} B B^{T} e^{A^{T} s} d s\right)^{-1} e^{A t_{s}} q(0), \\
t \in\left[0, t_{s}\right]
\end{gathered}
$$

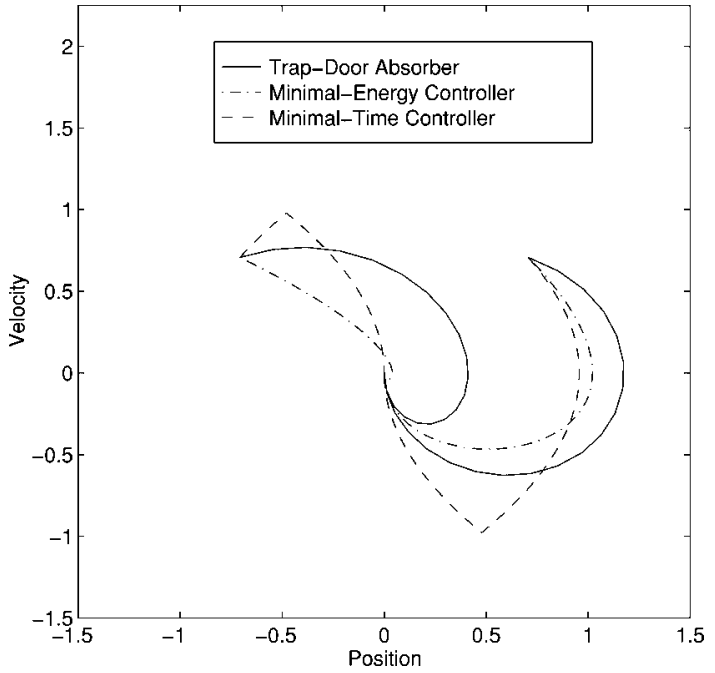

Fig. 3. Trajectories for two initial conditions plotted in phase space.

and in linear time-varying feedback form by

$$
\begin{gathered}
u(t)=-B^{T} e^{A^{T} t_{s}}\left(\int_{t}^{t_{s}} e^{A s} B B^{T} e^{A^{T} s} d s\right)^{-1} e^{A t_{s}} q(t), \\
t \in\left[0, t_{s}\right]
\end{gathered}
$$

which minimizes the cost functional $J=\int_{0}^{t s} u^{2}(t) d t$ and yields $q\left(t_{s}\right)=0$. For the double integrator (20) the control laws (25), (26) become, respectively

$$
\begin{aligned}
& u(t)=M\left(\frac{12 q_{10}}{t_{s}^{3}}+\frac{6 \dot{q}_{10}}{t_{s}^{2}}\right) t-M\left(\frac{6 q_{10}}{t_{s}^{2}}+\frac{4 \dot{q}_{10}}{t_{s}}\right), \\
& t \in\left[0, t_{s}\right] \\
& u(t)=\frac{-6 M}{\left(t_{s}-t\right)^{2}} q_{1}(t)-\frac{4 M}{t_{s}-t} \dot{q}_{1}(t), \quad t \in\left[0, t_{s}\right] .
\end{aligned}
$$

It can be shown that if the initial condition $q_{0}$ satisfies $q_{10}^{2}+\dot{q}_{10}^{2} \leq 1$, then

$$
|u(t)| \leq 2 M \sqrt{9+4 t_{s}^{2}} / t_{s}^{2}, \quad t \in\left[0, t_{s}\right] .
$$

To design the trap-door controller, we choose $n=p=0$ in (14), so that $k=4 K / 3$ and $m=4 M / 3$. $K$ will be chosen to satisfy a control amplitude constraint.

To compare these controllers, let $M=1$ and impose the control constraint $|u(t)| \leq 1, t \geq 0$. To satisfy this constraint for the minimal-time controller, we set $u_{\max }=1$, while for the minimal-energy controller with initial conditions inside the unit circle, we set $t_{s}=$ $3 \sqrt{2} \approx 4.24$. For the trap-door controller it follows that $m=4 / 3$, and we let $K=3 / 7$, so that $k=4 / 7$. With these values, (17) is equivalent to $|u(t)| \leq 1$, while $t_{s}=\sqrt{7} \pi / 2 \approx 4.16$.

In Figs. 3 and 4 , we choose initial conditions $q_{10}= \pm \sqrt{2} / 2, \dot{q}_{10}=$ $\sqrt{2} / 2$. The motion of $M$ is shown in Fig. 3, while the control is plotted in Fig. 4. Fig. 4 shows that the minimal-time control is piecewise constant with three discontinuities: switching on at $t=0$, switching sign, and switching off when $M$ reaches the origin. The minimal-energy and trap-door controllers each have two discontinuities: switching on at $t=0$ and switching off when $M$ reaches the origin.

Fig. 5 shows the tradeoff of control magnitude versus settling time, while Fig. 6 shows the tradeoff of control energy versus settling time for the initial condition $q_{10}=\sqrt{2} / 2$ and $\dot{q}_{10}=\sqrt{2} / 2$. For the minimal-time controller, values of $u_{\max }$ were chosen and the 


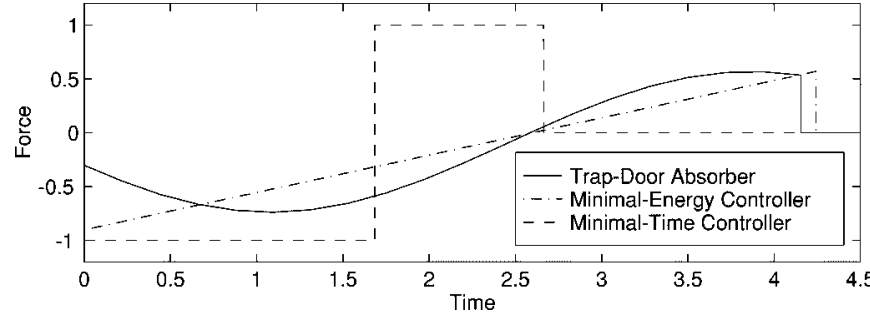

Fig. 4. Comparison of control histories with initial conditions $q_{10}=\sqrt{2} / 2$, $\dot{q}_{10}=\sqrt{2} / 2$.

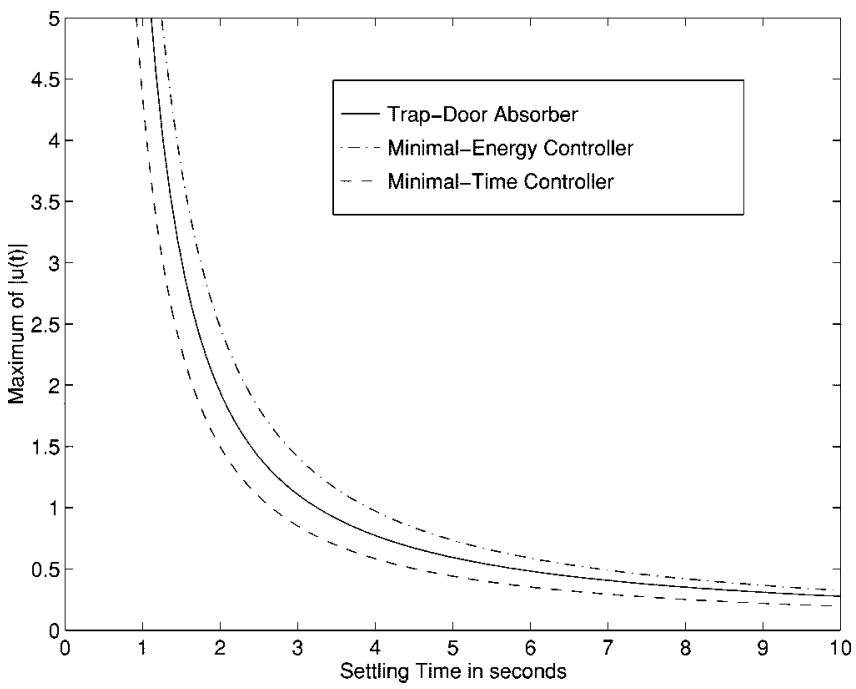

Fig. 5. Maximum control magnitude versus settling time for the initial condition $q_{10}=\sqrt{2} / 2, \dot{q}_{10}=\sqrt{2} / 2$.

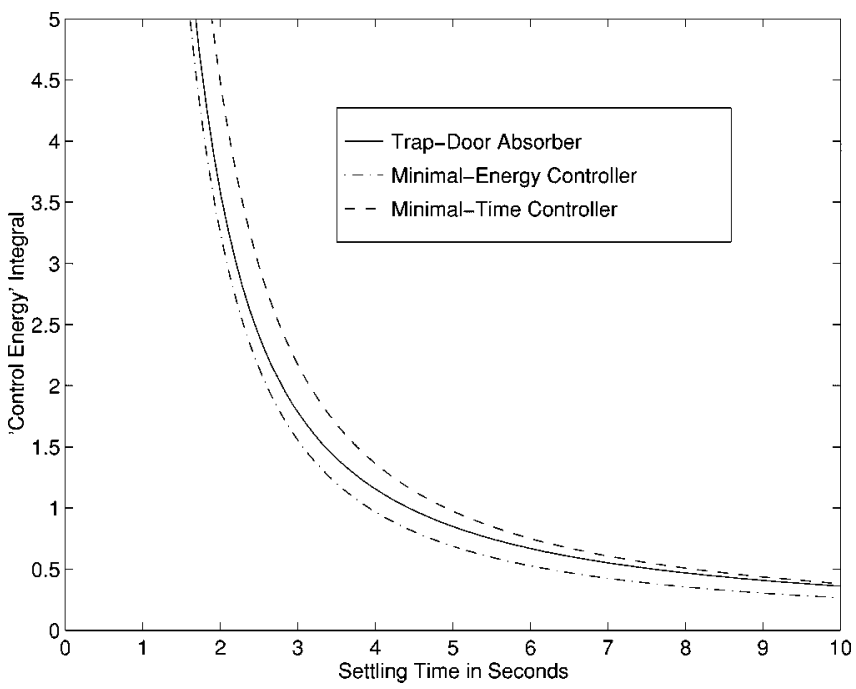

Fig. 6. $\int_{0}^{t_{s}} u^{2}(t) d t$ versus settling time for the initial condition $q_{10}=\sqrt{2} / 2$, $\dot{q}_{10}=\sqrt{2} / 2$.

corresponding settling times and energy integrals were computed. For the minimal-energy controller, values of the settling time $t_{s}$ were chosen and the resulting values of $u_{\max }$ and the energy integral $J$ were computed. Similarly, for the trap-door controller, values of $t_{s}$ were chosen, and the parameter $K$ was chosen according to Remark 3.2. The values of $u_{\max }$ and the energy integral $J$ were determined from the time history of the control. The simulations indicate that the trap-door controller has a better tradeoff of control magnitude versus settling time than the minimal-energy controller, and a better tradeoff of control energy versus settling time than the minimal-time controller.

For each controller, finite settling time performance is predicated on knowledge of $M$. Simulations indicate that for a $10 \%$ perturbation of $M$, the minimal-time controller chatters near the origin. Furthermore, the feedback form of the minimal-energy controller (28) exhibits control forces that approach infinity as time approaches the design settling time $t_{s}$

To implement the virtual trap-door absorber, we reset the states of the controller every $t_{s}$ seconds, rather than disconnecting the controller at time $t_{s}$; that is, as in [11], the states of the controller are periodically reassigned the values $q_{2}(t)=q_{1}(t), \dot{q}_{2}(t)=0$ with period $t_{s}$.

For $10 \%$ perturbation in $M$, the resetting virtual trap-door absorber control signal tends to decrease in amplitude with each resetting activity, while the states approach the origin. Due to the periodic resetting, the control signal exhibits regularly spaced discontinuities.

APPENDIX

PROOF OF THEOREM 3.1

Lemma 1: Let $a, b, f_{1}, f_{2} \in \mathbb{R}$, define $t_{s} \triangleq \pi(4 n+1) / 2 f_{1}$, and let

$$
\begin{gathered}
y(t)=a\left(\sin f_{1} t+\sin f_{2} t\right)+b\left(f_{2} \cos f_{1} t+f_{1} \cos f_{2} t\right), \\
t \geq 0 .
\end{gathered}
$$

If there exist integers $n, p \geq 0$ such that $f_{1} / f_{2}=(4 n+1) /(4 p+3)$, then $y\left(t_{s}\right)=\dot{y}\left(t_{s}\right)=0$.

Proof: Since $\sin f_{1} t_{s}=1, \cos f_{1} t_{s}=0, \sin f_{2} t_{s}=-1$, $\cos f_{2} t_{s}=0$, it follows that

$$
\begin{aligned}
y\left(t_{s}\right)= & a\left(\sin f_{1} t_{s}+\sin f_{2} t_{s}\right) \\
& +b\left(f_{2} \cos f_{1} t_{s}+f_{1} \cos f_{2} t_{s}\right)=0 \\
\dot{y}\left(t_{s}\right)= & a\left(f_{1} \cos f_{1} t_{s}+f_{2} \cos f_{2} t_{s}\right) \\
& -b f_{1} f_{2}\left(\sin f_{1} t_{s}+\sin f_{2} t_{s}\right)=0 .
\end{aligned}
$$

Proof of Theorem 3.1: Equation (14) yields

$$
\kappa=\frac{4(2(p-n)+1)^{2}}{(4 n+1)(4 p+3)}, \quad \omega_{a}=1 .
$$

Substituting (31) into (9) and using (13) yields

$$
\begin{aligned}
\omega & =1 / \Omega=\sqrt{\frac{4 n+1}{4 p+3}} \\
c_{1} & =-c_{2}=\frac{\Omega-\omega}{\Omega+\omega} \\
\frac{1}{2}\left(1+c_{1}\right) & =\frac{\Omega}{\Omega+\omega} \\
\frac{1}{2}\left(1-c_{1}\right) & =\frac{\omega}{\Omega+\omega} .
\end{aligned}
$$

Hence (12) becomes

$$
\begin{aligned}
x_{1}(\tau)= & \frac{x_{10}}{\omega+\Omega}[\Omega \cos \omega \tau+\omega \cos \Omega \tau] \\
& +\frac{\dot{x}_{10}}{\omega+\Omega}[\sin \omega \tau+\sin \Omega \tau]
\end{aligned}
$$

and it follows from Lemma 1 that $x_{1}\left(\tau_{s}\right)=0, \dot{x}_{1}\left(\tau_{s}\right)=0$, where

$$
\tau_{s}=\frac{\pi}{2} \frac{4 n+1}{\omega}=\frac{\pi}{2} \sqrt{(4 n+1)(4 p+3)}
$$

and thus $q_{1}\left(t_{s}\right)=0, \dot{q}_{1}\left(t_{s}\right)=0$, where $t_{s}$ is given by (16). 
To derive (17), the total energy $V(x)$ of the closed-loop system is

$$
V(x)=\frac{1}{2 K} x_{1}^{2}+\frac{1}{2 K} \dot{x}_{1}^{2}+\frac{1}{2 K} \kappa x_{r}^{2}+\frac{1}{2 K} \frac{\kappa}{\omega_{a}^{2}} \dot{x}_{2}^{2} .
$$

Since $\dot{V} \triangleq(\partial V / \partial x) \mathcal{A} x=0$, the total energy is constant; thus, with the initial condition (15)

$$
V(x(\tau)) \equiv V_{0} \triangleq \frac{1}{2 K} x_{10}^{2}+\frac{1}{2 K} \dot{x}_{10}^{2} .
$$

Hence

$$
\begin{aligned}
x_{1}^{2}(\tau)+\kappa x_{r}^{2}(\tau) & =2 K V_{0}-\dot{x}_{1}^{2}(\tau)-\frac{\kappa}{\omega_{a}^{2}} \dot{x}_{2}^{2}(\tau) \\
& \leq 2 K V_{0}, \quad \tau \geq 0 .
\end{aligned}
$$

To bound the control force, let $\lambda \in \mathbb{R}$ and form the Lagrangian

$$
\mathcal{L}\left(x_{1}, x_{r}, \lambda\right)=\kappa x_{r}-x_{1}+\lambda\left(x_{1}^{2}+\kappa x_{r}^{2}-2 K V_{0}\right) .
$$

Setting the partial derivatives of $\mathcal{L}$ to zero, yields $x_{r}=-x_{1}$. Hence (36) yields $(1+\kappa) x_{1}^{2} \leq 2 K V_{0}$, and thus $\left|x_{1}\right| \leq \sqrt{2 K V_{0} /(1+\kappa)}$. It follows from (6) that $|u|=(1+\kappa)\left|x_{1}\right| \leq \sqrt{2 K V_{0}(1+\kappa)}$, or, using (35), $|u| \leq \sqrt{(1+\kappa)\left(x_{10}^{2}+\dot{x}_{10}^{2}\right)}$. This yields (17).

\section{REFERENCES}

[1] S. V. Salehi and E. P. Ryan, "On optimal nonlinear feedback regulation of linear plants," IEEE Trans. Automat. Contr., vol. 27, pp. 1260-1264, 1982.

[2] V. T. Haimo, "Finite time controllers," SIAM J. Contr. Optim., vol. 24, pp. 760-770, 1986.

[3] K. Ichikawa, "Finite-settling-time control of continuous-time plants," Syst. Contr. Lett., vol. 9, pp. 341-343, 1987.

[4] S. Choura, "Design of finite time settling regulators for linear systems," Trans. ASME, vol. 116, pp. 602-608, 1994.

[5] J. M. Coron, "On the stabilization in finite time of locally controllable systems by means of continuous time-varying feedback law," SIAM J. Contr. Optim., vol. 33, pp. 804-833, 1995.

[6] S. P. Bhat and D. S. Bernstein, "Continuous finite-time stabilization of the translational and rotational double integrators," IEEE Trans. Automat. Contr., vol. 43, pp. 678-682, 1998.

[7] M. Athans and P. L. Falb, Optimal Control: An Introduction to the Theory and Its Applications. New York: McGraw-Hill, 1966.

[8] A. E. Bryson and Y.-C. Ho, Applied Optimal Control: Hemisphere, 1975.

[9] B. G. Korenov and L. M. Reznikov, Dynamic Vibration Absorbers: Theory and Technical Applications. New York: Wiley, 1993.

[10] J.-N. Juang and M. Phan, "Robust controller designs for second-order dynamic systems: A virtual passive approach," J. Guid. Contr. Dyn., vol. 15, pp. 1192-1198, 1992.

[11] R. T. Bupp, D. S. Bernstein, W. M. Haddad, and V. Chellaboina, "Resetting virtual absorbers for control," in Proc. American Control Conf., Albuquerque, NM, June 1997, pp. 2647-2651. also J. Vibr. Contr., vol. 6, pp. 61-83.

[12] R. T. Bupp and D. S. Bernstein, "Experimental implementation of integrator backstepping and passive nonlinear controllers on the RTAC testbed," Int. J. Robust Nonlinear Contl., vol. 8, pp. 435-457, 1998.

[13] K. L. Tuer, M. F. Golnaraghi, and D. Wang, "Toward a generalized regulation scheme for oscillatory systems via coupling effects," IEEE Trans. Automat. Contr., vol. 40, pp. 522-530, 1995.

\section{Optimal Control of a Queueing System with Heterogeneous Servers and Setup Costs}

\author{
Rein D. Nobel and Henk C. Tijms
}

\begin{abstract}
This paper considers a queueing model with batch Poisson input and two heterogeneous servers, where the service times are exponentially distributed. The faster server is always on, but the slower server is only used when the queue length exceeds a certain level. Activating the slower server involves fixed set-up costs. Also there are linear operating costs and linear holding costs. The class of two-level hysteretic control rules is considered. Rather than proving the overall average cost optimality of a hysteretic rule, the purpose of this paper is to develop a tailor-made policyiteration algorithm for computing the optimal switch-on and switch-off levels for the slower server. An embedding method is used that is generally applicable to structured Markovian control problems with an infinitely large state space.
\end{abstract}

Index Terms-Controlled queueing system, heterogeneous servers, Markov decision theory.

\section{INTRODUCTION}

Queueing models with heterogeneous servers play a role of increasing importance in many engineering areas, such as telecommunication and computer systems. Often we are faced with control problems in which servers (transmission lines) can be switched on and off, and costs are associated with the operation of servers and the presence of waiting jobs (messages). The motivation for studying such control problems comes from problems of dynamic routing in computer systems or communication networks. The basic control problem considered in this paper has batch Poisson input and two heterogeneous servers who share a common buffer of infinite capacity. The service times are exponentially distributed with different rates for the two servers. Service is nonpreemptive. The faster server is always activated, whereas the slower server can be both on and off. The slower server can only be switched on upon arrival of new jobs and it can only be switched off when it completes a service.

Lin and Kumar [3] studied this model under the criterion of minimizing the long-run average number of jobs in the system. Using dynamic programming arguments they proved the intuitively obvious result that the optimal control rule is of the threshold type, i.e., the slower server should be utilized only when the queue length exceeds a certain threshold value. Simpler proofs of the same result were later given in Koole [2] and Walrand [7], cf., also Viniotis and Ephremides [6]. Unlike these papers that focus on proving the structure of an optimal control rule, we focus on algorithmic aspects. This is done for a more general and more difficult problem involving fixed set-up costs for turning the slower server on. In addition to the switching costs we assume an operating cost at a constant rate for each unit of time the slower server is on. Further, it is assumed that there are linear holding costs for the jobs in the system. The goal is to compute a control rule that minimizes the long-run average cost per unit time. In view of the fixed set-up cost for turning the slower server on, we can no longer restrict ourselves to the threshold policies with a single critical level. An intuitively appealing rule in the new situation is the two-level hysteretic switching rule that

Manuscript received November 28, 1998; revised June 15, 1999. Recommended by Associate Editor, L. Dai. This work was supported in part by the INTAS 96-0828 research project.

The authors are with the Department of Econometrics, Vrije University, De Boelelaan 1105, 1081 HV Amsterdam, The Netherlands (e-mail: rnobel@econ.vu.nl; tijms@econ.vu.nl).

Publisher Item Identifier S 0018-9286(00)04088-5. 